\title{
LINEAR PROJECTIONS WHICH IMPLEMENT BALAYAGE IN FOURIER TRANSFORMS
}

\author{
GEORGE S. SHAPIRO
}

\begin{abstract}
Let $\Lambda$ be a closed and discrete or compact subset of a second countable LCA group $G$ and $E$ a subset of the dual group. Balayage is said to be possible for $(\Lambda, E)$ if for every finite measure $\mu$ on $G$ there is some measure $\nu$ on $\Lambda$ whose Fourier transform, $\hat{\nu}$, agrees on $E$ with $\hat{\mu}$.

If balayage is assumed possible just when $\mu$ is a point measure (with the norms of all the measures $\nu$ bounded by some constant), then there is a bounded linear projection, $B_{\Lambda}$, from the measures on $G$ onto those on $\Lambda$ with $\left(B_{\Lambda} \mu\right)^{\hat{}}=\hat{\mu}$ on $E$. An application is made to balayage in product groups.
\end{abstract}

Throughout $G$ denotes a second countable locally compact abelian group, $\Gamma$ its dual group and $\Lambda \subset G$ and $E \subset \Gamma$ closed sets. $M(G)$ is the convolution algebra of finite, regular, complex Borel measures on $G$ and $M(\Lambda)$ is the closed subspace of $M(G)$ consisting of those measures supported by $\Lambda$. If $\mu$ $\in M(G), \hat{\mu}$ denotes the (inverse) Fourier transform of $\mu$,

$$
\hat{\mu}(\gamma)=\int_{G}\langle\gamma, x\rangle d \mu(x) \quad \text { for } \gamma \in \Gamma .
$$

We write $\mu \sim \nu(E)$ to mean $\hat{\mu}(\gamma)=\hat{\nu}(\gamma)$ for all $\gamma \in E$.

Definition. Balayage is said to be possible for the pair $(\Lambda, E)$ if for every $\mu \in M(G)$ there is some $\nu \in M(\Lambda)$ with $\mu \sim \nu(E)$.

This situation was introduced and first studied by Beurling ([1], unfortunately unpublished, and [2]) especially for the case where $G$ is the real line. The two cases most studied, and which we deal with here, are those in which $\Lambda$ is either discrete or compact (in which case $E$ should have the other of these properties to make nontrivial balayage possible).

Our major result (which appeared in the author's dissertation [7]) is that, in the situation considered here, if balayage is possible for $(\Lambda, E)$, then there is a bounded linear projection of $M(G)$ onto $M(\Lambda)$ (of as small norm as is reasonable) which implements the balayage. Moreover, to obtain this conclusion from a measurable choice argument, we need assume only the possibility of (bounded) balayage of point measures, a fact which is crucial for an application we make to balayage in product groups. In a special case, namely when $\Lambda$ is discrete and $E$ is a compact convex set with interior in a Euclidean

Received by the editors April 26, 1976.

AMS (MOS) subject classifications (1970). Primary 43A25; Secondary 28A05.

Key words and phrases. Balayage in Fourier transforms, point measures, measurable choice, analytic set, weak- ${ }^{*}$ integral, bounded linear projection, balayage in product groups. 
space, Beurling [2] showed, by a variational technique, that the existence of a linear balayage operator follows from the possibility of bounded balayage of point measures. His proof also involves integrating a family of measures (somewhat as in Theorem 1) but does not produce a projection.

If balayage is possible for $(\Lambda, E)$, then an open mapping argument due to Beurling (see [8, §2]) shows the existence of a constant $K$ such that for every $\mu \in M(G)$ there is some $\nu \in M(\Lambda)$ with $\|\mu\| \leqslant K\|\nu\|$ and $\mu \sim \nu(E)$. The infimum of constants $K$ for which the above holds is denoted $K(\Lambda, E)$.

We write $\delta_{x}$ for the unit point mass at $x$ so that $\hat{\delta}_{x}(\gamma)=\langle\gamma, x\rangle$.

THEOREM 1. Let $G$ be a second countable locally compact abelian group and let $\Lambda \subset G$ be either closed and discrete or compact. Suppose that there is a constant $K$ such that for every $x \in G$ there is a measure $m(x) \in M(\Lambda)$ with $\|m(x)\|$ $\leqslant K$ and $m(x) \sim \delta_{x}(E)$. Then there is a bounded linear operator $B_{\Lambda}: M(G)$ $\rightarrow M(\Lambda)$ such that

1. $B_{\Lambda} \mu \sim \mu(E)$, for every $\mu \in M(G)$,

2. $B_{\Lambda} \mu=\mu$ for every $\mu \in M(\Lambda)$,

3. $\left\|B_{\Lambda}\right\| \leqslant K$.

In particular, $K(\Lambda, E) \leqslant K$.

Proof. The gist of the proof is to use a measurable choice result to replace the $m(x)$ by measures $\alpha(x)$ which depend measurably on $x$ and then to set $B_{\Lambda} \mu=\int_{G} \alpha(x) d \mu(x)$, the integral to be interpreted in the weak- ${ }^{*}$ sense.

We recall first the relevant topological facts.

Let $X$ and $Y$ be Polish spaces (separable spaces whose topologies can be defined by complete metrics). Suppose $Q \subset X \times Y$ is an analytic (Suslin) set whose projection, $\pi_{1}(Q)$, onto $X$ is all of $X$. Then there is a function $\alpha: X \rightarrow Y$ such that $(x, \alpha(x)) \in Q \forall x \in X$ and such that $\alpha^{-1}(U)$ is analytic for every open $U \subset Y$. In particular, $\alpha$ is measurable with respect to every regular Borel measure on $X$. (This is essentially von Neumann's original result [9, Lemma 5] as reformulated over the years. See also [3, Appendix V]. [6, Theorem 3] is a more modern and general formulation.)

Because $G$ is a second countable locally compact group, $G$ is a Polish space. We set $M_{K}(\Lambda)=\{\mu \in M(\Lambda):\|\mu\| \leqslant K\}$.

In the case where $\Lambda$ is discrete (and, hence, countable) we give $M_{K}(\Lambda)$ its norm topology. Then $M(\Lambda) \simeq l^{1}(\Lambda)$ is separable so that $M_{K}(\Lambda)$ is a Polish space. In this case, integrals are really sums but the integral notation is more suggestive and agrees better with the compact case.

In the case where $\Lambda$ is compact, we give $M_{K}(\Lambda)$ the restriction of the weak* topology of $M(\Lambda)$ regarded as the dual of $C(\Lambda)$ (the continuous complex functions on $\Lambda$ ). Since $\Lambda$ is separable metric, $C(\Lambda)$ is separable and, therefore, the weak- ${ }^{*}$ topology on $M_{K}(\Lambda)$ is metrizable [4, p. 426]. Since $M_{K}(\Lambda)$ is also weak- ${ }^{*}$ compact, it is a Polish space.

We deal with both cases together, indicating the requisite variations in the argument as they occur. 
We work in $G \times M_{K}(\Lambda)$. We set

$$
S=\left\{(x, \mu) \in G \times M_{K}(\Lambda): \mu \sim \delta_{x}(E)\right\} .
$$

$S$ is closed. Indeed, suppose $\left(x_{n}, \mu_{n}\right) \in S$ for $n=1,2, \ldots$ and

$$
\lim _{n \rightarrow \infty}\left(x_{n}, \mu_{n}\right)=(x, \mu) .
$$

Then $\lim _{n \rightarrow \infty} \mu_{n}=\mu$ in $M_{K}(\Lambda)$ which implies that $\lim _{n \rightarrow \infty} \hat{\mu}_{n}(\gamma)=\hat{\mu}(\gamma)$ for all $\gamma \in \Gamma$. (For the norm topology this is clear; for the weak- ${ }^{*}$ topology it follows because the restriction, $\gamma \mid \Lambda$, of $\gamma$ to $\Lambda$ is in $C(\Lambda)$.) Thus, for $\gamma \in E$,

$$
\hat{\mu}(\gamma)=\lim _{n \rightarrow \infty} \hat{\mu}_{n}(\gamma)=\lim _{n \rightarrow \infty}\left\langle\gamma, x_{n}\right\rangle=\langle\gamma, x\rangle=\hat{\delta}_{x}(\gamma)
$$

Hence, $(x, \mu) \in S$.

$T=\Lambda \times M_{K}(\Lambda)$ is closed.

$U=\left\{\left(\lambda, \delta_{\lambda}\right): \lambda \in \Lambda\right\}$ is closed. (In the discrete case because $\lambda \neq \lambda^{\prime}$ implies that $\left\|\delta_{\lambda}-\delta_{\lambda^{\prime}}\right\|=2$ and in the compact case because $U$ is the image of the compact set $\Lambda$ under the map $\lambda \mapsto\left(\lambda, \delta_{\lambda}\right)$.)

We set $Q=S-(T-U)$ (where - denotes relative complementation). Since every closed set in a metric space is a $G_{\delta}$, it is easily checked that $Q$ is an $F_{\sigma}$ and, hence, an analytic set. By construction, $Q$ has the following properties:

(a) $(x, \mu) \in Q \Rightarrow \mu \sim \delta_{x}(E)$.

(b) $\left(\lambda, \delta_{\lambda}\right) \in Q$ for all $\lambda \in \Lambda$.

(c) $(x, m(x)) \in Q$ for all $x \in G-\Lambda$.

(d) $(\lambda, \mu) \in Q$ and $\lambda \in \Lambda \Rightarrow \mu=\delta_{\lambda}$.

In particular, (b) and (c) imply that $\pi_{1}(Q)=G$. We now employ the measurable choice result quoted above to obtain a mapping $\alpha: G \rightarrow M_{K}(\Lambda)$ such that $(x, \alpha(x)) \in Q$ for all $x$ and such that $\alpha$ is measurable with respect to every $\mu \in M(G)$ (which property we shall henceforth refer to simply as measurability).

Clearly $\|\alpha(x)\| \leqslant K$. From (a) above we deduce that $\alpha(x) \sim \delta_{x}(E)$ for all $x \in G$, while from (d) we see that $\alpha(\lambda)=\delta_{\lambda}$ if $\lambda \in \Lambda$.

We shall of ten write $\langle\phi, \mu\rangle$ for $\int \phi d \mu$.

$C_{0}(\Lambda)$ denotes the set of continuous functions on $\Lambda$ vanishing at infinity (=C( $=$ in the compact case). If $\phi \in C_{0}(\Lambda)$, then the map $\nu \mapsto\langle\phi, \nu\rangle$ is continuous as a map from $M_{K}(\Lambda)$ (with either topology) to the complex numbers. Thus, $x \mapsto\langle\phi, \alpha(x)\rangle$ is measurable for every such $\phi$. This permits us to define, for $\mu \in M(G), B_{\Lambda} \mu=\int \alpha(x) d \mu(x)$, the integral being interpreted in the weak- * sense. More precisely, $B_{\Lambda} \mu$ is defined by the condition that

$$
\left\langle\phi, B_{\Lambda} \mu\right\rangle=\int_{G}\langle\phi, \alpha(x)\rangle d \mu(x)
$$

for all $\phi \in C_{0}(\Lambda)$. Then $B_{\Lambda} \mu \in M(\Lambda)$ and standard arguments show that $B_{\Lambda}$ is linear and that $\left\|B_{\Lambda}\right\| \leqslant K$. 
If $\mu \in M(\Lambda)$, then for each $\phi \in C_{0}(\Lambda)$, we have

$$
\begin{aligned}
\left\langle\phi, B_{\Lambda} \mu\right\rangle & =\int_{\Lambda}\langle\phi, \alpha(\lambda)\rangle d \mu(\lambda)=\int_{\Lambda}\left\langle\phi, \delta_{\lambda}\right\rangle d \mu(\lambda) \\
& =\int_{\Lambda} \phi(\lambda) d \mu(\lambda)=\langle\phi, \mu\rangle .
\end{aligned}
$$

Thus, $B_{\Lambda} \mu=\mu$.

In the compact case, if $\gamma \in E$ then $\gamma \mid \Lambda \in C_{0}(\Lambda)$ and

$$
\begin{aligned}
\left(B_{\Lambda} \mu\right)^{\hat{}} & =\left\langle\gamma \mid \Lambda, B_{\Lambda} \mu\right\rangle=\int_{G}\langle\gamma \mid \Lambda, \alpha(x)\rangle d \mu(x) \\
& =\int_{G}(\alpha(x))^{\hat{\gamma}}(\gamma) d \mu(x)=\int_{G}\langle\gamma, x\rangle d \mu(x)=\hat{\mu}(\gamma)
\end{aligned}
$$

completing the proof of the theorem in this case.

For the discrete case, we remark that every $\phi \in C(\Lambda)$ can be expressed as a pointwise limit, $\phi=\lim _{n \rightarrow \infty} \phi_{n}$, where $\phi_{n} \in C_{0}(\Lambda)$ and $\left\|\phi_{n}\right\|_{\infty} \leqslant\|\phi\|_{\infty}$. Then two applications of the dominated convergence theorem show that (1) holds also for $\phi \in C(\Lambda)$. If $\gamma \in E$, then $\gamma \mid \Lambda \in C(\Lambda)$ and the argument of the previous paragraph now applies in this case also, completing the proof of the theorem.

REMARKS. (1) If $\Lambda$ is compact, then so is $M_{K}(\Lambda)$ and now the fact that $Q$ is an $F_{\sigma}$ implies that $x \mapsto \alpha(x)$ may be chosen to be Borel measurable. (Use [5, Theorem 1] and the fact that a map between Polish spaces which has a Borel graph is Borel measurable.)

(2) Theorem 1 can be extended, in an ad hoc fashion, to certain other types of $\Lambda$, for example, to $\Lambda$ the disjoint union of a discrete closed set and a compact set. What is required is the existence of a "reasonable" Polish, or at least Suslin, topology $\mathcal{T}$ on $M_{K}(\Lambda)$ no weaker than the weak- * topology and such that $\mathcal{T}$-convergence implies pointwise convergence of Fourier transforms on $E$. For general closed $\Lambda$, however, $M_{K}(\Lambda)$ will not be second countable.

THEOREM 2. Let $G_{1}$ and $G_{2}$ be second countable LCA groups with dual groups $\Gamma_{1}$ and $\Gamma_{2}$. Regard $\Gamma_{1} \times \Gamma_{2}$ as the dual of $G_{1} \times G_{2}$. Suppose $\Lambda_{i} \subset G_{i}(i=1,2)$ are either both closed and discrete or both compact and let $E_{i} \subset \Gamma_{i}$. If balayage is possible for $\left(\Lambda_{i}, E_{i}\right), i=1,2$, then balayage is possible for $\left(\Lambda_{1} \times \Lambda_{2}, E_{1} \times E_{2}\right)$ and

$$
K\left(\Lambda_{1} \times \Lambda_{2}, E_{1} \times E_{2}\right) \leqslant K\left(\Lambda_{1}, E_{1}\right) K\left(\Lambda_{2}, E_{2}\right) .
$$

Proof. Let $\varepsilon>0$ and suppose $\left(x_{1}, x_{2}\right) \in G_{1} \times G_{2}$. Then, for $i=1,2$, there is some $\mu_{i} \in M\left(\Lambda_{i}\right)$ with $\left\|\mu_{i}\right\| \leqslant K\left(\Lambda_{i}, E_{i}\right)+\varepsilon$ and $\mu_{i} \sim \delta_{x_{i}}\left(E_{i}\right)$. Let $\mu=\mu_{1} \times \mu_{2}$ be the product measure. Then $\|\mu\| \leqslant\left\|\mu_{1}\right\|\left\|\mu_{2}\right\|$. If $\left(\gamma_{1}, \gamma_{2}\right)$ $\in E_{1} \times E_{2}$, a simple computation gives

$$
\begin{aligned}
\hat{\mu}\left(\gamma_{1}, \gamma_{2}\right) & =\hat{\mu}_{1}\left(\gamma_{1}\right) \hat{\mu}_{2}\left(\gamma_{2}\right)=\left\langle\gamma_{1}, x_{1}\right\rangle\left\langle\gamma_{2}, x_{2}\right\rangle \\
& =\left\langle\left(\gamma_{1}, \gamma_{2}\right),\left(x_{1}, x_{2}\right)\right\rangle .
\end{aligned}
$$

Thus, $\mu \sim \delta_{\left(x_{1}, x_{2}\right)}\left(E_{1} \times E_{2}\right)$. 
Since $\Lambda_{1} \times \Lambda_{2}$ is either closed and discrete or else compact, Theorem 1 now implies that

$$
K\left(\Lambda_{1} \times \Lambda_{2}, E_{1} \times E_{2}\right) \leqslant\left(K\left(\Lambda_{1}, E_{1}\right)+\varepsilon\right)\left(K\left(\Lambda_{2}, E_{2}\right)+\varepsilon\right) .
$$

Since $\varepsilon$ is arbitrary, the result follows.

\section{BIBLIOGRAPHY}

1. A. Beurling, On balayage of measures in Fourier transforms, Seminar notes, Institute for Advanced Study, Princeton, N.J., 1959-1960 (unpublished).

2. - Local harmonic analysis with some applications to differential operators, Annual Science Conference Proceedings, I (1962-1964), Belfer Graduate School of Science, Yeshiva Univ., New York, 1966, pp. 109-125.

3. J. Dixmier, Les algèbres d'opérateurs dans l'espace hilbertien (algèbres de von Neumann), Cahiers scientifiques, fasc. 25, Gauthier-Villars, Paris, 1957. MR 20 \# 1234.

4. N. Dunford and J. T. Schwartz, Linear operators. Part I, Interscience, New York, 1958. MR 22 \#8302.

5. C. A. Rogers and R. C. Willmott, On the uniformization of sets in topological spaces, Acta Math. 120 (1968), 1-52. MR 38 \#6014.

6. M.-F. Sainte-Beuve, On the extension of von Neumann-Aumann's theorem, J. Functional Analysis 17 (1974), 112-129.

7. G. S. Shapiro, Some aspects of balayage of Fourier transforms, Dissertation, Harvard Univ., 1973.

8. - Balayage in Fourier transforms: general results, perturbation and balayage with sparse frequencies, Trans. Amer. Math. Soc. (to appear).

9. J. von Neumann, On rings of operators. Reduction theory, Ann. of Math. (2) 50 (1949), 401-485. MR 10, 548.

Department of Mathematics, City University of New York, Brooklyn College, BROOKLYN, NEW YoRK 11210 